\title{
Extension of locally pseudocompact group actions
}

Received: June 22, 2000; in final form: May 22, 2001

Published online: June 11, 2002 - ㄷ Springer-Verlag 2002

\begin{abstract}
It is shown that: (1) any action of a Moscow group $G$ on a first countable, Dieudonné complete (in particular, on a metrizable) space $X$ can uniquely be extended to an action of the Dieudonné completion $\gamma G$ on $X$, (2) any action of a locally pseudocompact topological group $G$ on a $b_{f}$-space (in particular, on a first countable space) $X$ can uniquely be extended to an action of the Weil completion $\bar{G}$ on the Dieudonné completion $\gamma X$ of $X$. As a consequence, we obtain that, for each locally pseudocompact topological group $G$, every $G$-space with the $b_{f}$-property admits an equivariant embedding into a compact Hausdorff $G$-space. Furthermore, for each pseudocompact group $G$, every metrizable $G$-space has a $G$-invariant metric compatible with its topology. We also give a direct construction of such an invariant metric.
\end{abstract}

Mathematics Subject Classification (2000). 22F05, 54H15, 54D35

Key words. (locally) pseudocompact group - action - equivariant - compactification

By a $G$-space we mean a triple $(G, X, \alpha)$, where $G$ is a topological group, $X$ is a topological space, and $\alpha: G \times X \rightarrow X$ is a continuous action. For basic information on $G$-spaces, see [20], [23]. As in [12], we will call a topological group $G$ a $V$-group if every Tychonov $G$-space can equivariantly be embedded into a compact Hausdorff $G$-space.

A famous theorem of Jan de Vries [22] states that every locally compact Hausdorff group $G$ is a $V$-group (see also [13] for $G$, a compact Lie group, and [1] for $G$, a compact Hausdorff group). Megrelishvili [11], answering a question of de Vries [21], constructed a continuous action $\alpha$ of a separable, complete metrizable group $G$ on $J\left(\aleph_{0}\right)$, the so-called metrizable hedgehog of countable spininess [9, p. 314], such that $\left(G, J\left(\aleph_{0}\right), \alpha\right)$ does not admit an equivariant embedding into a compact Hausdorff $G$-space. It is an open question whether there exist non-locally compact $V$-groups. Recently Megrelishvili and Scarr [12] proved that each $\aleph_{0^{-}}$ bounded $V$-group is locally precompact (a subgroup of a locally compact group). In this connection they asked: is every locally precompact (non-locally compact) group a $V$-group?

S. Antonyan: Departamento de Matemáticas, Facultad de Ciencias UNAM, Circuito Exterior, C.U. 04510 México D.F. (México), e-mail: antonyan@servidor . unam. mx

M. Sanchis: Departament de Matemàtiques, Universitat Jaume I, Campus del Riu Sec, s/n, 12071 Castelló (Spain), e-mail: sanchis@mat.uji.es

* The first author was supported in part by PAPIIT grant IN-105800 of UNAM 
In the present paper we show that any action of a Moscow group on a metrizable space $X$ can uniquely be extended to an action of the Dieudonné completion $\gamma G$ on $X$. Moreover, as it is stated in Theorem 5, each locally pseudocompact group is a "semi- $V$-group" in the following sense: if $G$ is a locally pseudocompact group and $X$ is a $G$-space with the $b_{f}$-property (for instance, if $X$ is first countable) then $X$ can equivariantly be embedded into a compact Hausdorff $G$-space. In this case every continuous action $G \times X \rightarrow X$ can be uniquely extended to a continuous action $\gamma G \times \gamma X \rightarrow \gamma X$, where $\gamma Z$ stands for the Dieudonné topological completion of $Z$ (Theorem 4).

We achieve the aim by applying some results on the distribution of the functor of the Dieudonné topological completion. Incidentally we also prove a new result on this (Theorem 2). For $G$, a pseudocompact group, Theorem 6 yields the existence of a $G$-invariant metric on each metrizable $G$-space (Corollary 4$)$. We also give a direct construction of such an invariant metric (Theorem 6). The reader can find other related results in the text.

Throughout the paper all spaces are assumed to be Tychonov (i.e., completely regular and Hausdorff) and all groups are Hausdorff. We recall that a space $X$ is said to be Moscow [3] if, for each open subset $U$ of $X$, the closure of $U$ is the union of a family of $G_{\delta}$-subsets of $X$.

A space $X$ is called pseudocompact if every real-valued function on $X$ is bounded. A topological group is called locally pseudocompact if the identity has a pseudocompact neighborhood (equivalently: if the identity has a local basis of pseudocompact neighborhoods). Locally pseudocompact groups are locally precompact, so each such group $G$ is densely embedded in an essentially unique locally compact group $\bar{G}$ which is named its Weil completion. We say that a space $X$ is Dieudonné complete if $X$ is homeomorphic to a closed subspace of a product of metrizable spaces. It is known that for every space $X$ there exists a Dieudonné complete space $\gamma X$, unique up to a homeomorphism, which leaves $X$ pointwise fixed, in which $X$ is dense and every continuous function from $X$ into a Dieudonné complete space admits a continuous extension on $\gamma X . \gamma X$ is called the Dieudonné topological completion of $X$. Recall that a subset $Y$ of a space $X$ is called $G_{\delta}$-dense (respectively, $C$-embedded) in $X$ if it intersects every nonempty $G_{\delta}$-subset of $X$ (respectively, every continuous real-valued function on $Y$ admits a continuous extension to $X)$. It is an easy consequence of Theorem 8.7 in [10] and 8.5.8 (b) in [9] that $\gamma X$ is the unique Dieudonné complete space, up to homeomorphisms which leave $X$ pointwise fixed, in which $X$ is $G_{\delta}$-dense and $C$-embedded.

Notice that infinite products of locally compact groups are Moscow (see [16]) and, since dense subsets of Moscow spaces are Moscow, infinite products of locally precompact groups (in particular of locally pseudocompact groups) are also Moscow.

The notion of a Moscow space is a very important tool in the study of the Dieudonné completion. For instance, in [5], answering an interesting question of Pestov and Tkačenko [14], [18], the notion of Moscow space is used to prove that there exist topological groups whose Dieudonné completion is not a topological group. 
On the other hand, Comfort and Trigos-Arrieta proved in [8] that the Dieudonné completion of a locally pseudocompact group $G$ coincides with its Weil completion $\bar{G}$. The second author improved this result by showing that the Dieudonné completion of an arbitrary product of locally pseudocompact groups is the product of their Weil completions [15] and, consequently, it is a topological group.

Our first result combines these properties and a new result on the distribution of the functor of the Dieudonné topological completion in order to study continuous actions of arbitrary Moscow groups on metrizable spaces.

The following result obtained by Uspenskii [19], which generalizes a previous one of Tkačenko [17] in the realm of $\kappa$-normal spaces, establishes one of the most important properties of Moscow spaces:

Theorem 1 (Tkačenko-Uspenskiı̌'s Theorem). Every $G_{\delta}$-dense subset of a Moscow space is $C$-embedded.

Theorem 2. Let $G$ be a Moscow group. Then $\gamma(G \times X)=\gamma G \times X$ holds for each first countable, Dieudonné complete (in particular, metrizable) space $X$.

Proof. According to Theorems 1.1 and 1.2 in [5], $\gamma G$ is a Moscow group. Since the product of a Moscow space and a first countable space is Moscow, we have that $G \times X$ is a $G_{\delta}$-dense subspace of the Moscow space $\gamma G \times X$. By the Tkačenko-Uspenskii Theorem above, $G \times X$ is $C$-embedded in the Dieudonné complete space $\gamma G \times X$. It then follows that $\gamma(G \times X)=\gamma G \times X$.

Lemma 1. Let $G$ be a topological group and let $X$ be a space. If $\gamma(G \times X)=$ $\gamma G \times \gamma X$, then every continuous action $\alpha: G \times X \rightarrow X$ admits a unique extension to a continuous action $\tilde{\alpha}: \gamma G \times \gamma X \rightarrow \gamma X$.

Proof. The map $\alpha: G \times X \rightarrow X$ admits a continuous extension $\sigma: \gamma(G \times X) \rightarrow$ $\gamma X$. By hypothesis, one thus has a continuous extension $\sigma: \gamma G \times \gamma X \rightarrow \gamma X$ of the map $\alpha$. We claim that $\sigma$ is an action. Indeed, as $\alpha$ is an action, the map $\sigma(1, \cdot)$ coincides with the identity of $\gamma X$ on its dense subset $X$. Consequently it is the identity map of $\gamma X$. Next we define the maps $f_{1}, f_{2}: \gamma G \times \gamma G \times \gamma X \rightarrow \gamma X$, according to the role

$$
f\left(g_{1}, g_{2}, x\right)=\sigma\left(g_{1}, \sigma\left(g_{2}, x\right)\right) \text { and } h\left(g_{1}, g_{2}, x\right)=\sigma\left(g_{1} g_{2}, x\right) .
$$

As $\alpha=\left.\sigma\right|_{G \times X}$ is an action, $f$ and $h$ coincide on $G \times G \times X$. As $G \times G \times X$ is dense in $\gamma G \times \gamma G \times \gamma X$, we conclude that $f=h$, which means exactly that $\sigma$ is an action. The uniqueness of $\tilde{\alpha}$ is evident.

Theorem 3. Let $G$ be a Moscow group. Then every continuous action $\alpha: G \times X$ $\rightarrow X$ on a first countable, Dieudonné complete space $X$ admits a unique extension to a continuous action $\tilde{\alpha}: \gamma G \times X \rightarrow X$.

Proof. According to Theorem 2 and Lemma 1, the action $\alpha$ admits a unique continuous extension $\tilde{\alpha}: \gamma G \times \gamma X \rightarrow \gamma X$. Since $X$ is Dieudonné complete, $\gamma X=X$, and the proof is complete. 
As every metrizable space is first countable and Dieudonné complete, from Theorem 3 we get the following:

Corollary 1. Let $G$ be a Moscow group. Then every continuous action $\alpha: G \times X$ $\rightarrow X$ on a metrizable space $X$ admits a unique extension to a continuous action $\tilde{\alpha}: \gamma G \times X \rightarrow X$.

We turn now to the case $G$, a locally pseudocompact group. It seems interesting to point out that local pseudocompactness is carried over finite products (see Theorem 4.1 in [15]). Several definitions are in order. A subset $B$ of a space $X$ is said to be relatively pseudocompact (in $X$ ) if every continuous real-valued function on $X$ is bounded on $B$. So, a space is pseudocompact if it is relatively pseudocompact in itself. A function $f$ from a space $X$ into a space $Y$ is called $b_{f}$-continuous if the restriction of $f$ to each relatively pseudocompact subset of $X$ admits a continuous extension to all of $X$. A space such that every $b_{f}$-continuous real-valued function is continuous is called a $b_{f}$-space (or a space with the $b_{f}$-property). $b_{f}$-spaces arise in several realms of general topology and functional analysis, notably when studying compactness in function spaces [5] and in the problem of the distribution of the Dieudonné topological completion [6]. Locally pseudocompact spaces and $k_{r}$-spaces (spaces $X$ such that a real-valued function on $X$ is continuous whenever its restriction to every compact subset is continuous) are examples of $b_{f}$-spaces. So first countable (then metrizable) spaces are $b_{f}$-spaces. Notice that every Dieudonné complete $b_{f}$-space is a (Dieudonné complete) $k_{r}$-space.

Theorem 4. Let $G$ be a locally pseudocompact group. Then every continuous action $\alpha: G \times X \rightarrow X$ on a $b_{f}$-space $X$ admits a unique extension to a continuous action $\tilde{\alpha}: \gamma G \times \gamma X \rightarrow \gamma X$.

Proof. By Theorem 4.6 in [15], the equality $\gamma(G \times X)=\gamma G \times \gamma X$ holds. Then apply Lemma 1.

Let $f: X \rightarrow Y$ be a $G$-map of $G$-spaces with the $b_{f}$-property. Denote by $f_{\gamma}: \gamma X \rightarrow \gamma Y$ the unique continuous extension of $f$. We observe that then $f_{\gamma}$ is a $\gamma G$-equivariant map. Indeed, define the following two continuous maps $F, \Phi: \gamma G \times \gamma X \rightarrow \gamma Y$ according the rules $F(t)=f_{\gamma}(t x)$ and $\Phi(t)=t f_{\gamma}(x)$, where $t \in \gamma G$ and $x \in \gamma X$. Since $f$ is $G$-equivariant, the maps $F$ and $\Phi$ coincide on the dense subset $G \times X$ of $\gamma G \times \gamma X$. Consequently, $F=\Phi$, which means that $f_{\gamma}(t x)=$ $t f_{\gamma}(x)$ for all $t \in \gamma G$ and $x \in X$. Thus $f_{\gamma}$ is $\gamma G$-equivariant. Now compare to every $G$-space $(G, X, \alpha)$ with $X$ a $b_{f}$-space, the $\gamma G$-space $(\gamma G, \gamma X, \tilde{\alpha})$, and to every $G$-map $f: X \rightarrow Y$, the $\gamma G$-map $f_{\gamma}: \gamma X \rightarrow \gamma Y$. This functorial correspondence in a definite sense reduces the investigation of locally pseudocompact (respectively, pseudocompact) group actions on $b_{f}$-spaces to the investigation of locally compact (respectively, compact) group actions on Dieudonné complete spaces.

Theorem 5. Let $G$ be a locally pseudocompact group. Then every $G$-space $X$ with the $b_{f}$-property admits an equivariant embedding into a compact $G$-space.

Proof. Since $\gamma G=\bar{G}$ is a locally compact group [8], the claim is immediate from the above mentioned result of de Vries [22] and from Theorem 4. 
As $\gamma X=X$ for each Dieudonné complete space $X$, from Theorem 4 we then get the following:

Corollary 2. Let $G$ be a locally pseudocompact group. Then every continuous action $\alpha: G \times X \rightarrow X$ on a Dieudonné complete $b_{f}$-space $X$ admits unique extension to a continuous action $\tilde{\alpha}: \gamma G \times X \rightarrow X$.

Corollary 3. Let $G$ be a locally pseudocompact group (respectively, pseudocompact) group and $X$ be a first countable, Dieudonné complete $G$-space. Then for each point $x \in X$, the stabilizer $G_{x}=\{g \in G: g x=x\}$ is a closed locally pseudocompact (respectively, pseudocompact) subgroup of $G$. Moreover, $\gamma\left(G_{x}\right)=(\gamma G)_{x}$ holds true.

Proof. Consider the continuous map $f: G \rightarrow X$ defined by $f(g)=g x, g \in G$. Then $G_{x}=f^{-1}(x)$. As points are closed, $G_{\delta}$ sets in $X$ we conclude that $G_{x}$ is a closed, $G_{\delta}$ subgroup of $G$. Now it follows from [8, Theorem 2.11] (respectively, [8, Remark 2.7]) that $G_{x}$ is locally pseudocompact (respectively, pseudocompact). By Corollary 2 , the $G$-action on $X$ can uniquely be extended to a $\gamma G$-action on $X$. To obtain the equality $\gamma\left(G_{x}\right)=(\gamma G)_{x}$, we observe that $c l_{\gamma G} G_{x}$ is a locally compact group, where the locally pseudocompact group $G_{x}$ is $G_{\delta}$-dense and, consequently, $c l_{\gamma G} G_{x}=\overline{G_{x}}=\gamma\left(G_{x}\right)$. We show that $(\gamma G)_{x}=c l_{\gamma G} G_{x}$. Indeed, as above, each stabilizer $(\gamma G)_{x}$ is a closed $G_{\delta}$ set in $\gamma G$. Now if $W$ is an open subset in $\gamma G$ then $(\gamma G)_{x} \cap W$ is also a $G_{\delta}$ set in $\gamma G$. As $G$ is $G_{\delta}$-dense in $\bar{G}=\gamma G$ [8, Remark 3.4], we conclude that $(\gamma G)_{x} \cap$ $W \cap G \neq \emptyset$. But $G_{x}=(\gamma G)_{x} \cap G$, and hence, we get $\left(\gamma G_{x}\right) \cap W \cap G_{x} \neq \emptyset$.

Corollary 4. Let $G$ be a pseudocompact group. Then every metrizable $G$-space $X$ admits a $G$-invariant metric compatible with its topology.

Proof. By Corollary 2, the action $\alpha: G \times X \rightarrow X$ can be extended to an action $\tilde{\alpha}: \gamma G \times X \rightarrow X$. As $G$ is pseudocompact, one has $\gamma G=\beta G$ [7]. By compactness of the group $\beta G$, there is a $\beta G$-invariant metric $\rho$ on $X$, compatible with its topology [13, Proposition 1.1.12] (see also [2] for an alternate proof). In particular, $\rho$ is also $G$-invariant.

In the rest of the paper we shall present yet another (direct) proof of Corollary 4. In fact the following more precise result holds true:

Theorem 6. Let $G$ be a pseudocompact topological group. Let $\rho$ be a metric, compatible with the topology of a metrizable $G$-space $X$. Then the formula $d(x, y)=\operatorname{Sup}\{\rho(g x, g y): g \in G\}$ defines an invariant metric, also compatible with the topology of $X$.

The proof depends upon two lemmas.

In what follows for spaces $X$ and $Y$ we will denote by $C(X, Y)$ the space of all continuous functions $f: X \rightarrow Y$, equipped with the compact-open topology.

Lemma 2. Let $X$ be a space containing a dense $\sigma$-compact subset and $Y$ be a metrizable space. Then every pseudocompact subset in $C(X, Y)$ is metrizable. 
Proof. Let $X=\overline{\bigcup_{n=1}^{\infty} X_{n}}$, where each $X_{n}$ is a compact subset of $X$. For every $n \geq 1$, let $p_{n}: C(X, Y) \rightarrow C\left(X_{n}, Y\right)$ be the restriction map, which compares to each $f \in C(X, Y)$ its restriction $\left.f\right|_{X_{n}}$. Since each $p_{n}$ is continuous, so is their diagonal product $p: C(X, Y) \rightarrow \prod_{n=1}^{\infty} C\left(X_{n}, Y\right)$. If $p(f)=p(\varphi)$ for $f, \varphi \in$ $C(X, Y)$, then the maps $f$ and $\varphi$ coincide on the dense subset $\bigcup_{n=1}^{\infty} X_{n}$, and since $X$ is Hausdorff, they must coincide on the whole space $X$. Consequently, $f=\varphi$, showing that $p$ is injective. By the compactness of $X_{n}, C\left(X_{n}, Y\right)$ is metrizable, and hence, the countable product $\prod_{n=1}^{\infty} C\left(X_{n}, Y\right)$ is metrizable too. Consequently, for every pseudocompact subset $K$ of $C(X, Y)$ the image $p(K)$, being a pseudocompact subset of the metrizable space $\prod_{n=1}^{\infty} C\left(X_{n}, Y\right)$, is a compact metrizable space. Thus the restriction $\left.p\right|_{K}: K \rightarrow p(K)$ is a condensation on the metrizable compactum $p(K)$. Due to the pseudocompactness of $K$, this condensation is a homeomorphism (see [4, Lemma 5.6]). Therefore $K$ is metrizable.

The following important result is well known for $G$, a compact group (see e.g., [13]):

Lemma 3. Let a pseudocompact group $G$ act effectively on a metrizable space $X$, which possesses a dense $\sigma$-compact subset. Then $G$ is metrizable (and hence, compact).

Proof. Let $\alpha: G \times X \rightarrow X$ be an effective action. The map $\alpha^{*}: G \rightarrow C(X, X)$ defined by $\alpha^{*}(g)(x)=\alpha(g, x), g \in G, x \in X$ is continuous [9, p. 244]. As the image $\alpha^{*}(G)$ is pseudocompact, Lemma 2 yields its metrizability. By the effectiveness of the action $\alpha$, the map $\alpha^{*}: G \rightarrow \alpha^{*}(G)$ is a condensation, and since $G$ is pseudocompact, this condensation is a homeomorphism (see [4, Lemma 5.10]). Consequently $G$ is metrizable.

Proof of Theorem 6. Evidently $d$ is an invariant metric, and $\rho(x, y) \leq d(x, y)$ for all $x, y \in X$. Let us show that the $\rho$-convergence in $X$ implies the $d$-convergence. Assume that the contrary is true. Then there exist a point $x \in X$ and a sequence $\left\{x_{n}\right\} \subseteq X$ such that $\rho\left(x_{n}, x\right) \rightsquigarrow 0$, while $d\left(x_{n}, x\right)$ does not converge to zero. Then for some $\varepsilon>0$ and for some subsequence $\left\{y_{k}\right\} \subseteq\left\{x_{n}\right\}$, the inequalities $d\left(y_{k}, x\right) \geq \varepsilon$, $k=1,2, \ldots$ hold. But then there is a sequence $\left\{g_{k}\right\} \subseteq G$ such that

$$
\rho\left(g_{k} y_{k}, g_{k} x\right)>\varepsilon / 2, \quad k=1,2, \ldots
$$

Consider the $G$-space $Z=G(x) \cup \bigcup_{n=1}^{\infty} G\left(y_{k}\right)$, where $G(a)$ denotes the orbit of a point $a \in X$. Let $N$ be the kernel of the action of $G$ on $Z$, i.e., $N=\{g \in G$ : $g z=z$ for all $z \in Z\}$. Then the quotient group $G / N$ acts (by the same rule) effectively on $Z$. As $Z$ is $\sigma$-compact and $G / N$ is pseudocompact, Lemma 3 yields that $G / N$ is a metrizable compact space. Let $g_{k}^{*}=p\left(g_{k}\right)$ where $p: G \rightarrow G / N$ is the projection. By the compactness of $G / N$, the sequence $\left\{g_{k}^{*}\right\}$ has a limit point $g^{*} \in G / N$. As the action of $G / N$ on $Z$ is continuous, there are neighborhoods $H \subseteq G / N$ and $U \subseteq Z$ of points $g^{*}$ and $x$, respectively, such that

$$
\rho\left(h^{*} y, g^{*} x\right)<\varepsilon / 4, \quad h^{*} \in H, y \in U .
$$


As $\rho\left(y_{k}, x\right) \rightsquigarrow 0$ then there is a positive integer $r$ such that $y_{k} \in U$ for all $k \geq r$. Since $g^{*}$ is a limit point of $\left\{g_{k}\right\}$ one has $g_{m}^{*} \in H$ for some $m>r$. But then (6) yields that $\rho\left(g_{m}^{*} y_{m}, g^{*} x\right)<\varepsilon / 4$ and $\rho\left(g_{m}^{*} x, g^{*} x\right)<\varepsilon / 4$. Consequently, $\rho\left(g_{m}^{*} y_{m}, g_{m}^{*} x\right)<$ $\varepsilon / 2$, which contradicts to (5) because $g_{m}^{*} y_{m}=g_{m} y_{m}$ and $g_{m}^{*} x=g_{m} x$.

Beginning from the previous results, the following specific questions seem worthy of study:

Question 1. Is every locally pseudocompact group a $V$-group?

Question 2. Let $G$ be an arbitrary Moscow group. Does every continuous action of $G$ on a Dieudonné complete $b_{f}$-space $X$ admit a unique continuous extension to an action of $\gamma G$ on $X$ ?

Acknowledgements. The authors owe a debt of gratitude to the referee for turning our attention to Moscow spaces, in particular, to Theorem 2 which improved the first version by the authors.

\section{References}

1. Antonyan, S.A.: A new proof of the existence of compact $G$-extensions. Comment. Math. Univ. Carol. 22(4), 761-772 (1981). In Russian

2. Antonian, S.A.: Equivariant embedding into G-AR's. Glasnik Matem. 22(1), 503-533 (1987)

3. Arkhangel'skiř, A.: Functional tightness, $Q$-spaces, and $\tau$-embeddings. Comment. Math. Univ. Carol. 24(1), 105-120 (1983)

4. Arkhangel'skiř, A.: Function spaces in the topology of pointwise convergence, and compact sets. Russian Math. Surv. 39(5), 11-50 (1984)

5. Arkhangel'skiř, A.: Moscow spaces, Pestov-Tkaěnko problem and $C$-embeddings. Comment. Math. Univ. Carol. 41(3), 585-595 (2000)

6. Buchwalter, H.: Produit topologique, produit tensoriel et $c$-replétion. Bull. Soc. Math. France Suppl., Mém. 31-32, 51-71 (1972)

7. Comfort, W.W., Ross, K.: Pseudocompactness and uniform continuity in topological groups. Pac. J. Math. 16(3), 483-496 (1966)

8. Comfort, W.W., Trigos-Arieta, F.J.: Locally pseudocompact topological groups. Top. Appl. 62, 263-280 (1995)

9. Engelking, R.: General Topology. PWN 1977

10. Gillman, L., Jerison, M.: Rings of Continuous Functions. New York: Van Nostrand Reinhold 1960

11. Megrelishvili, M.: A Tychonoff $G$-space which has no compact extensions. Russian Math. Surv. 43(2), 177-178 (1988)

12. Megrelishvili, M., Scarr, T.: Constructing Tychonoff $G$-spaces which are not $G$-Tychonoff. Top. Appl. 86, 69-81 (1998)

13. Palais, R.: The classification of $G$-spaces, Memoirs AMS 36 (1960)

14. Pestov, V.G., Thačenko, M.G.: Unsolved Problem in Topological Algebra. Moldova, Kishinev, "Shtiinca": Academy of Science 1985

15. Sanchis, M.: Continuous functions on locally pseudocompact groups. Top. Appl. 86, 5-23 (1998)

16. Shchepin, E.V.: On $\kappa$-metrizable spaces. Izv. AN SSSR, Ser. Matem. 43(2), 442-478 (1979)

17. Tkačenko, M.G.: The notion of $o$-tightness and $C$-embedded subspaces of products. Top. Appl. 15(1), 93-98 (1983) 
18. Tkačenko, M.G.: Subgroups, quotient groups, and products of $R$-factorizable groups. Top. Proc. 16, 201-231 (1991)

19. Uspenskiǔ, V.V.: Topological groups and Dugundji compact spaces. Math. USSR-Sb. 67(2), 555-580 (1990)

20. de Vries, J.: Topological Transformation groups I. Mathematical Centre Tracts 65. Amsterdam: Mathematish Centrum 1975

21. de Vries, J.: Can every Tychonoff $G$-space equivariantly be embedded in a compact Hausdorff $G$-space? Afd. Zuiverse Wisk. 36 (1975)

22. de Vries, J.: On the existence of $G$-compactifications. Bull. Acad. Polon. Sci. Ser. Math. 26, 275-280 (1978)

23. de Vries, J.: Elements of Topological Dynamics. Mathematics and its Applications. Dortrecht-Boston-London: Kluwer Academic Publ. 1993 